\title{
Pre-operative Chlorhexidine mouth rinses reduce the incidence of dry socket
}

\section{What is the best method to prevent alveolar osteitis when patients undergo dental extraction?}

\begin{abstract}
Hedstrom L, Sjogren P.
Effect estimates and methodological quality of randomized controlled trials about prevention of alveolar osteitis following tooth extraction: a systematic review. Oral Surg Oral Med Oral Pathol Oral Radiol Endod 2007; 103:8-15
\end{abstract}

Data sources Medline and the Cochrane library databases were searched and additional studies located by scrutinising publications obtained.

Study selection Studies included in analysis were randomised controlled trails (RCT) about prevention of alveolar osteitis (AO) that were written in English, French, German or any of the Nordic languages (Danish, Finnish, Icelandic, Norwegian, Swedish). Duplicate publications, those with flawed data and RCT that generally addressed postoperative complications were excluded.

Data extraction and synthesis The RCT were quality assessed using the Jadad scale and then categorised and tabulated, according to the main test interventions, to the following domains: antibiotics trials; chlorhexidine trials; trials of PEPH (an antifibrinolytic-active propylic ester of $p$-hydrobenzoic acid); and other trials including factorial trials (combined interventions) and factorial test groups of RCT belonging to any of the aforementioned domains.

Results A total of 90 publications were identified, from which 32 RCT were included, from 12 different countries, covering the time period 1971-2005. An adequate method of random allocation was reported in $47 \%$ of the RCT. Eight different RCT assessed different antibiotic regimens. Tetracycline trials revealed the greatest preventive effects on $\mathrm{AO}$, with absolute risk reductions (ARR) ranging from $12-31 \%$ and numbers needed to treat (NNT) ranging from three to eight treated individuals.

The effect of chlorhexidine rinses for prevention of $A O$ was studied in five RCT with ARR ranging from 3-25\% (NNT, four to 36 treatments). Overall, evidence about chlorhexidine rinses on the prevention of $\mathrm{AO}$ was inconclusive, but the available data indicate that $0.12 \%$ chlorhexidine rinsing pre-operatively and 7 days postoperatively reduces the frequency of AO following surgical removal of lower third molars. Similarly, evidence for the effectiveness of the antifibrinolytic agent PEPH (considered in three trials) was inconclusive, as was that for the other 18 trials agents.

Conclusions Local treatment with tetracycline, and also $0.12 \%$ chlorhexidine rinsing pre-operatively and 7 days postoperatively, seem to have significant and clinically relevant preventive effect on $A O$ following surgical removal of lower third molars.

Address for correspondence: Petteri Sjögren, Public Dental Services, Box 1254, SE43218 Varberg, Sweden. E-mail: igps@tele2.se.

\section{Commentary}

This is an impressive systematic review in terms of the numbers of RCT identified and included. On the face of it, given the frequency with which dry socket occurs and its acute nature, the literature should abound with high quality trials. Trials of interventions designed to prevent disease have their own particular problems, not least that sample sizes need to be very large unless the disease or complication is very frequent. Very surprisingly, therefore, in the 32 trials reviewed, only four included a power calculation for sample size! A further difficulty is that definitions of dry socket varied considerably. For example, few attempts were made in the component trials to establish whether one of the classic signs of dry socket, loss of the socket blood clot, was present. This may seem over-critical until one realises that many trials focus on complications after third molar surgery where dry socket is much less common than other wound infection. Indeed, after many surgical third molar extractions, the 'socket' is no longer in its presurgical form and may even have been eliminated altogether.

Although the findings suggest that local tetracycline is the most effective treatment, sample sizes in trials where other antibiotics have been used were often insufficient and the authors are right to be cautious about recommending its routine use, especially in view of the potential for hypersensitivity reactions and systemic toxicity. These risks may be small at an individual level, but may be high at population level if application is recommended as part of such a high-volume procedure as dental extraction, and cannot be in the interests of overall health. The results of this review do, however, strengthen the case for rinsing with chlorhexidine pre-operatively and for the first few days postoperatively. As has been stated in an excellent metaanalysis, previously reviewed in Evidence-based Dentistry ${ }^{1}$, "further studies using a standard definition for dry socket, and stratification of patients by gender, difficulty of extraction, smoking status etc., are needed to determine the best chlorhexidine (regimen)" ${ }^{2}$

\section{Practice point}

This systematic review provides clinically significant evidence that repeated chlorhexidine mouth rinses, beginning pre-operatively, reduce the incidence of dry socket.

\section{Jonathan Shepherd}

Department of Oral Surgery, Oral Medicine and Oral Pathology, Cardiff University School of Dentistry, Cardiff, Wales, UK

1. Shepherd J. Rinsing with chlorhexidine may reduce incidence of dry socket after third molar surgery. Evid Based Dent. 2005;6(2):36

2. Caso A, Hung LK, Beirne OR. Prevention of alveolar osteitis with chlorhexidine: a meta-analytic review. Oral Surg Oral Med Oral Pathol Oral Radiol Endod 2005 99:155-159. 